\title{
CHARACTERIZATION OF HEAT GENERATION OF GOLD NANORODS RADIATED BY A NIR LASER FOR CLINICAL CANCER THERAPY
}

\author{
Ji Yong Bae ${ }^{1}$, Chanbae Jung ${ }^{1}$, Ki-Soo Chang ${ }^{1}$ and Ki-Hwan Nam ${ }^{1+*}$ \\ ${ }^{1}$ Optical Instrumentation Development Team, Korea Basic Science Institute, Daejeon 34133, Rep. of Korea \\ +*Presenting and Corresponding Author: namkihwan@kbsi.re.kr
}

\begin{abstract}
Photothermal therapy provides a strong potential in treatment of tumors through the ability of gold nanoparticles to target destructive heat preferentially to tumor regions. And yet, Clinical application of the thermal therapies has not accomplished due to insufficient processes of the heating methods and temperature measuring techniques leading to low reproducibility of such treatment. In this study, we demonstrate various three-dimensional artificial tissue platforms to characterize the heating method and to control the generated heat in the tissue used for a superficial cancer model using gold nanorods (GNRs) and near-infrared (NIR, $808 \mathrm{~nm}$ ) laser. GNRs have been shown to be the large absorption cross sections generating localized photothermal effects and hyperthermic effects on destructive consequences in the cell dynamics and survival rates. In particular, the GNRs were coated with polystyrenesulfonate (PSS, CTAB-free) to increase their stability and biocompatibility, and to enable the effectiveness for cancer therapy. The 3D tissue platforms had a $2 \mathrm{~mm}$ wide half dome considered as where cancers cells are thought to be, covered with $20 \mu \mathrm{m}$ thick polymer film to confine the GNRs in the room. Various biomaterials were tested to mimic mechanical and optical properties of the soft tissue those found in vivo. To investigate the photothermal effect of GNRs in the tissue, three variables (the depth of the tissue, the amount of GNRs, and light density) were controlled to characterize the heat generation and distribution of GNRs created in the tissue.
\end{abstract}

KEYWORDS: Photothermal cancer therapy, gold nanorods, 808nm laser, 3D tissue platform, human decellularized adipose tissue, porcine skin gel

\section{ACKNOWLEDGMENT}

This work was supported by Korea Basic Science Institute grant (2B0605), and Ministry of Science, ICT, and Future Planning fund (PG2017065). 